\title{
Alterstice
}

Revue internationale de la recherche interculturelle

International Journal of Intercultural Research

Revista International de la Investigacion Intercultural

\section{Conceptions successives de la mobilité dans la formation initiale des enseignants de la Pädagogische Hochschule Bern : diversification des pratiques à travers l'expérience de la diversité francophone?}

\section{Jésabel Robin}

Volume 7, numéro 2, 2017

Diversité(s) au coeur des politiques et des pratiques : entre l'Europe et l'Afrique

URI : https://id.erudit.org/iderudit/1052569ar

DOI : https://doi.org/10.7202/1052569ar

Aller au sommaire du numéro

Éditeur(s)

Alterstice

ISSN

1923-919X (numérique)

Découvrir la revue

Citer cet article

Robin, J. (2017). Conceptions successives de la mobilité dans la formation initiale des enseignants de la Pädagogische Hochschule Bern : diversification des pratiques à travers l'expérience de la diversité francophone? Alterstice, 7(2), 55-65. https://doi.org/10.7202/1052569ar
Résumé de l'article

Nous allons étudier les conceptions institutionnelles successives des expériences de mobilité étudiante dans la formation initiale germanophone des enseignants du primaire de la Pädagogische Hochschule Bern (PHBern) de Berne en Suisse alémanique. En effet, à en juger par l'évolution des programmes en lien avec la mobilité ainsi que par les récits qu'en font les acteurs, il semble que de nouvelles dynamiques se mettent en place dans le rapport à l'espace francophone. À travers les expériences de mobilité de ces étudiants au contact de la diversité francophone, voit-on émerger de nouveaux rapports au français et de nouvelles pratiques professionnelles au sein de l'institution? 


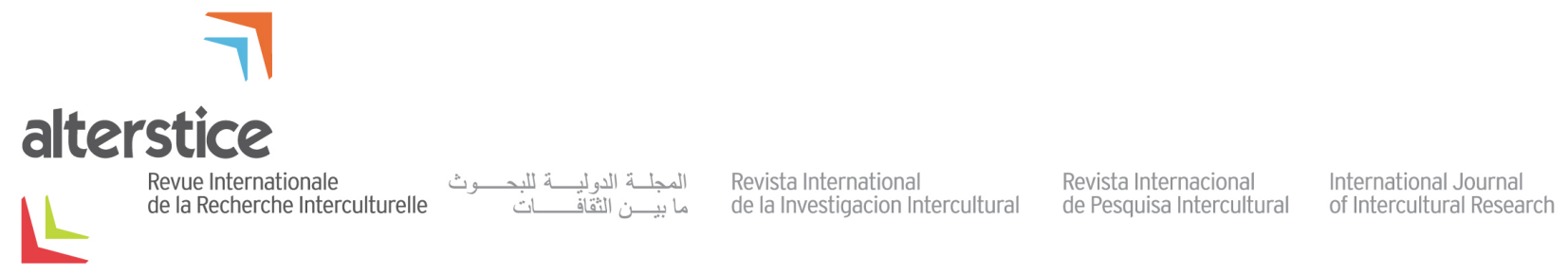

ARTICLE THÉMATIQUE

\section{Conceptions successives de la mobilité dans la formation initiale des enseignants de la Pädagogische Hochschule Bern : diversification des pratiques à travers l'expérience de la diversité francophone?}

Jésabel Robin ${ }^{1}$

\section{Résumé}

Nous allons étudier les conceptions institutionnelles successives des expériences de mobilité étudiante dans la formation initiale germanophone des enseignants du primaire de la Pädagogische Hochschule Bern (PHBern) de Berne en Suisse alémanique. En effet, à en juger par l'évolution des programmes en lien avec la mobilité ainsi que par les récits qu'en font les acteurs, il semble que de nouvelles dynamiques se mettent en place dans le rapport à l'espace francophone. À travers les expériences de mobilité de ces étudiants au contact de la diversité francophone, voit-on émerger de nouveaux rapports au français et de nouvelles pratiques professionnelles au sein de l'institution?

\section{Rattachement de l'auteure \\ ${ }^{1}$ Pädagogische Hochschule Bern (PHBern), Berne, Suisse}

\section{Correspondance}

jesabel.robin@phbern.ch

\section{Mots clés}

formation des enseignants, didactique de la mobilité, dispositif institutionnel, récits d'expériences, diversité des pratiques

\section{Pour citer cet article}

Robin, J. (2017). Conceptions successives de la mobilité dans la formation initiale des enseignants de la Pädagogische Hochschule Bern : diversification des pratiques à travers l'expérience de la diversité francophone? Alterstice, $7(2)$, 55-66. 


\section{Introduction : conceptions successives de la mobilité}

Ancrés en didactique des langues et des cultures (DLC), nos précédents travaux ont porté sur le rapport au français des étudiants de la Pädagogische Hochschule Bern (PHBern) en Suisse alémanique (Robin, 2014a). L'analyse qualitative de divers types de récits d'expériences a notamment permis de confirmer que de nombreux futurs enseignants germanophones du primaire et enseignants de FLE (français langue étrangère) présentent des résistances à l'encontre d'un de leurs futurs objets d'enseignement. À la peur et aux souffrances qui sont bien souvent liées à l'apprentissage de cette langue dite "étrangère » dans les plans d'études viennent s'ajouter des exigences institutionnelles élevées en termes de compétences à atteindre. Toutefois, nous avons constaté que les expériences de mobilité en région francophone imposées par la formation initiale constituaient un lieu de remédiation potentielle du rapport au français. Or la conception institutionnelle de la mobilité a évolué ces dernières années, si l'on en juge par les nouveaux programmes proposés - aussi bien dans le cadre de la formation initiale en français que dans l'encadrement des semestres d'échange universitaire ou même encore avec les projets de formations bilingues. Que sous-tendent ces conceptions successives de la mobilité étudiante et quels en sont les fondements théoriques? Cet article porte sur les enjeux institutionnels qui se cachent derrière la mobilité et leurs potentiels effets sur les acteurs de la mobilité en formation initiale.

\section{Présentation du terrain : de futurs enseignants de FLE malgré eux et mobilité imposée}

Nous allons nous pencher sur le cas de l'Institut für Vorschulstufe und Primarstufe (IVP) de la Pädagogische Hochschule Bern (PHBern). Situé en ville de Berne, cet institut forme les futurs enseignants de l'école primaire au sein de la Haute École Pédagogique germanophone du canton de Berne ${ }^{1}$. À la fois capitale d'un pays constitutionnellement plurilingue (allemand-français-italien-romanche) et d'un canton officiellement bilingue (allemand-français), Berne, bien que située à quelques kilomètres de la partie francophone de la Suisse, est une commune exclusivement germanophone. II nous faut préciser que la Suisse est une "mosaïque linguistique " (Windisch, 2007, p. 13) extrêmement complexe. Mises à part les rares communes officiellement bilingues, son fonctionnement a plus trait à la juxtaposition de communes à fonctionnement monolingue qu'à un véritable fonctionnement plurilingue. Entre langues officielles et langues nationales, plurilinguisme constitutionnel et principe de territorialité, le statut accordé aux langues induit des rapports de force qui se traduisent par des rapports ambigus, voire conflictuels, entre les différentes communautés linguistiques ${ }^{2}$.

La formation initiale au métier d'enseignant du primaire dispensée à la PHBern vaut pour 180 ECTS ${ }^{3}$ et est sanctionnée par un diplôme de Bachelor ${ }^{4}$. Les enseignants du primaire étant exclusivement des généralistes dans le canton de Berne, ils sont formés à enseigner toutes les matières obligatoires du programme scolaire. Le français y est la première langue " étrangère " obligatoire (littéralement Fremdsprache dans les plans d'études) enseignée dès la $3^{\mathrm{e}}$ année d'école primaire à hauteur de 3 leçons au minimum par semaine. Les enseignants du primaire étant ainsi tous de potentiels enseignants de FLE, le FLE constitue une matière obligatoire de la formation à l'IVP, à hauteur de 5 à 8 ECTS. Cette formation est composée des cours de langue et de didactique et est validée par des travaux orientés vers la future pratique professionnelle de l'enseignement du français au niveau primaire: préparations de matériel pédagogique, préparations détaillées de leçons suivant le manuel en vigueur, simulation de séquences didactiques, correction de productions d'élèves, mise en place de scénarios d'échanges, etc. Au niveau pratique, l'enseignement du français dans une classe alémanique fait également l'objet de l'un des cinq stages pratiques obligatoires de la formation. Enfin, les étudiants sont tenus d'organiser au cours de leurs trois années de formation un séjour de mobilité en territoire francophone (en Suisse ou à l'étranger). La conception institutionnelle de ce "séjour » ne cesse de faire peau neuve.

\footnotetext{
${ }^{1}$ La PHBern dispose également d'autres instituts de formation initiale : pour les futurs enseignants du secondaire 1 (équivalent du collège), du secondaire 2 (équivalent du lycée) et de l'enseignement spécialisé.

${ }^{2}$ Le terme de communauté linguistique est issu de la Constitution de 1999 qui le définit comme : "population ou groupe d'individus partageant de fait la même langue ".

${ }^{3}$ European Credits Transfer System : 1 ECTS correspond à environ 30 heures de travail.

${ }^{4}$ L'équivalent du diplôme de licence en France.
} 


\section{La mobilité pour la mobilité : progrès linguistiques et autres conceptions opportunistes de la diversité}

Depuis la fin des années 1980, on ne compte plus les recommandations institutionnelles et les discours politiques en faveur des échanges scolaires et universitaires. La mobilité devient omniprésente et l'on en fait l'apologie dans tous les milieux éducatifs. Si les institutions universitaires qui proposent à leurs étudiants des séjours de mobilité allant du simple stage pratique aux semestres, parfois consécutifs, d'échange s'appuient sur les recommandations du Conseil de l'Europe, rares sont cependant celles qui semblent prendre le temps de penser cette mobilité en termes éducatifs et d'élaborer des objectifs spécifiques en fonction de leurs objectifs de formation. II semble en effet commun de croire que le fait de se déplacer permet automatiquement de doter les acteurs de toutes sortes de compétences : linguistiques, personnelles, interculturelles, etc. Or il est depuis longtemps établi en DLC que le contact avec l'altérité ne signifie pas forcément le développement des compétences interculturelles, la mobilité étudiante pouvant servir au contraire à renforcer les stéréotypes plus ou moins valorisants sur la langue et ses locuteurs (Zarate, 1993).

Nous avons analysé des récits d'étudiants de la PHBern dans le cadre de nos précédents travaux (Robin, 2015b) ${ }^{5}$. Les objectifs du séjour linguistique (littéralement Sprachaufenthalt) jusqu'en 2011 étaient : " l'amélioration des compétences linguistiques " et "l'approfondissement des connaissances culturelles " ${ }^{6}$. On laisse ainsi penser que quatre semaines de séjour dit linguistique permettront de progresser dans les compétences linguistiques: l'institution se décharge de - externalise - la formation en langue. Elle " se reconnaît donc des limites en terme de formation, auxquelles la mobilité semble être la solution quasi "magique" » (Robin, 2013b, p. 95). Lorsque l'on sait que le Conseil de l'Europe recommande environ 200 heures de travail pour passer d'un niveau du Cadre Européen Commun de Référence pour les langues (CECR) à un autre, on comprend que les déceptions de la part des étudiants à leur retour soient fortes (quand bien même ils auraient assidûment fréquenté des cours de langue).

La consigne présuppose que les étudiants font forcément des progrès au niveau des compétences langagières et interculturelles. Elle exclut complètement les potentiels replis identitaires, les doutes, etc. Le séjour est donc perçu de la part de l'institution comme ne pouvant être que positif. (Robin, 2013a, p. 122)

Enfin, si le séjour linguistique est validé sur présentation d'un rapport, celui-ci n'est ni évalué, ni intégré par la suite dans la formation.

Les étudiants sont tenus d'organiser eux-mêmes leur séjour en dehors de leur temps d'études, c'est-à-dire sur leur temps personnel. Cela contraint en soi la durée du séjour linguistique puisque, de fait, en dehors du temps universitaire, les étudiants n'ont guère plus de quatre semaines de vacances en été. L'occupation est libre (travail, cours dans une école de langue, etc.) mais il est obligatoire de loger sur place et de le prouver. De nombreux étudiants travaillent en parallèle à leurs études ou sont parents et ne peuvent se permettre de " prendre des vacances " auprès de leur patron ou de s'absenter de la maison pour effectuer ce séjour. L'aspect financier et les contraintes familiales sont difficilement compatibles avec l'exigence institutionnelle. II est, de plus, difficile de qualifier ou typifier le séjour linguistique : il dure entre quatre semaines et six mois pour ceux qui choisissent d'effectuer un semestre d'échange universitaire. En marge du dispositif académique pourtant commanditaire, il prend alors les formes les plus diverses, en lien ou non avec la future profession: chantier humanitaire en Afrique francophone, travail à la réception d'un camping dans le sud de la France, cours intensifs dans une école de langue à Lausanne, jeune fille au pair dans une famille de paysans en Suisse romande, semestre d'échange universitaire en Belgique, etc. On peut donc parler de mobilité institutionnelle à " géométrie variable " (Gohard-Radenkovic, 2004, p. 59).

Diverses pratiques de contournement, instrumentalisation ou autres du séjour linguistique se mettent en place, allant même jusqu'au mensonge éhonté. "Les modalités institutionnelles du séjour sont peu encourageantes, [...] et plusieurs de nos informateurs nous ont confié en entretien qu'ils n'avaient aucune envie d'effectuer leur séjour

\footnotetext{
${ }^{5}$ La référence bibliographique indiquée renseignera au besoin sur la nature de ces divers récits, ainsi que sur les méthodes de recueil et d'analyse employées.

${ }^{6}$ Il s'agit d'une traduction personnelle du document officiel, disponible en ligne jusqu'en 2011 et rédigé en allemand.

${ }^{7}$ Le CECR définit six niveaux, qui servent de référence à la PHBern pour mesurer les compétences linguistiques des étudiants.
} 
de mobilité avant leur départ " (Robin, 2015a, p. 208). La mise en œuvre même de la mobilité est discutable: l'authenticité par exemple d'une simple lettre rédigée par de prétendues connaissances établies en Suisse romande certifiant que l'étudiant a travaillé dans la famille pendant quatre semaines ne pouvant être vérifiée, on comprend à quel point il est aisé de tricher et à quel point la pratique des certificats de complaisance est répandue.

Pourtant, malgré les résistances au départ et le jour peu attrayant sous lequel il se présente, le séjour se révèle parfois être un lieu-pivot pour la remédiation du rapport au français. C'est ce que révèle l'analyse de divers récits d'étudiants, notamment leurs « carte(s) de langue(s) et de mobilité(s) » (Robin, 2014b).

Pour nos informateurs en ayant fait l'expérience, l'articulation entre expériences de mobilité en milieu francophone et expériences d'enseignement en milieu francophone semble extrêmement féconde. C'est un lieu-pivot au niveau de leurs représentations et, plus largement, il s'agit d'un tournant réflexif au niveau de la construction d'une pratique professionnelle. (Robin, 2015b, p. 413)

La mobilité peut avoir pour effet de proposer un lieu de remédiation, voire de réconciliation, du rapport au français. Nos précédents travaux ont ainsi montré que les stages pratiques réalisés en milieux francophones peuvent avoir un impact fort. Exposés au triple déplacement (linguistique, culturel, professionnel) des pratiques de l'enseignement, les étudiants font alors des expériences d'enseignement qui restent fondamentales pour leurs propres pratiques d'enseignement en construction. La mobilité, les représentations des étudiants et les pratiques institutionnelles de la mobilité se révèlent liées et méritent d'être articulées conceptuellement.

\section{D'une conception linguistique à une conception socio-anthropologique : se préparer à la diversité en milieu professionnel}

La tentation est toujours la même, pour tous les acteurs sociaux, et les enseignants n'échappent pas plus que les autres à ce destin-là : celle de prendre sa position pour la position, c'est-à-dire la seule possible. On est toujours tenté de considérer que le monde est objectivement identique à la manière dont on le voit. (Porcher, 1997, p. 25)

Les travaux en DLC sont unanimes : c'est avant le départ et " dans l'établissement d'origine » (Anquetil, 2012) que l'encadrement doit commencer. Certains proposent un travail en deux étapes avec une préparation et une réflexion, d'autres proposent en outre d'accompagner le séjour. La réflexion post-mobilité " dont l'objectif est de faire conscientiser les compétences acquises et les transformations vécues pendant ce séjour d'immersion " (Gohard-Radenkovic, 2009a, p. 145) semble déterminante pour faire fructifier le séjour. Ce processus réflexif constitue d'ailleurs le réinvestissement qui permet le travail sur l'habitus plébiscité dans la formation (Vasseur, 2008 , p. 183) et les approches autobiographiques s'y prêtent particulièrement. Rien ne semble d'ailleurs plus logique dans le cadre d'une formation universitaire que de mettre en place ce genre de "démarche réflexive caractéristique de l'enseignement supérieur 》 (Anquetil, 2008, p. 234).

Ainsi, la contrainte de mobilité en région francophone a fait peau neuve et la formation FLE est complétée par un dispositif obligatoire en trois parties: préparation avant, accompagnement pendant et analyses après les expériences de mobilité. Ce ne sont désormais plus les compétences linguistiques qui sont visées: "II s'agit d'effectuer des expériences articulant différents types de mobilité: mobilité géographique, mobilité linguistique, mobilité culturelle et mobilité professionnelle $\|^{8}$. S'appuyant sur les approches socio-anthropologiques, l'institution n'exige plus de "séjour linguistique" mais des "expériences de mobilité ". De "séjour", l'on passe à " expériences », de " linguistique ", l'on passe à « mobilité ». II s'agit d'un véritable changement de paradigme. Ces expériences prennent dorénavant le plus souvent la forme d'un stage pratique, à visées professionnelles donc, de plusieurs semaines dans une école francophone suisse ${ }^{9}$. Il s'agit d'être à même de remettre en question des habitudes professionnelles ancrées, ce qui « nécessite des compétences de réflexivité qui ne sont pas innées mais qui se développent » (Auger, Molinié, Goï et Guillaumin, 2011, p. 181). Il s'agit donc d'éduquer à la mobilité, c'est-à-dire de doter les étudiants d'outils leur permettant de transcender leurs expériences et « d'accompagner

\footnotetext{
${ }^{8}$ Il s'agit d'une traduction personnelle du document officiel, disponible en ligne et rédigé en allemand (voir sitographie).

${ }^{9}$ Le semestre d'échange universitaire avec le bureau des Relations Internationales reste une option possible mais ne concerne par année que 10 à 15 étudiants, contre environ 200 qui choisissent dorénavant le stage pratique.
} 
les étudiants à réfléchir à la manière dont leurs représentations à propos des langues et de l'apprentissage des langues sont empreintes de leurs "cultures d'enseignement-apprentissage" » (Auger, Molinié, Goï et Guillaumin, 2011, p. 180).

Le fait de s'immerger dans un autre système éducatif incite les étudiants à remettre en question leurs croyances professionnelles et à entrer dans des pratiques de classe différentes de celles qui ont cours en Suisse alémanique. La diversité dans ce cas correspond aux pratiques scolaires, et notamment aux potentielles différences entre celles de la Suisse alémanique (considérées comme " évidentes " et "normales " par les étudiants) et celle de la Suisse francophone (qui peuvent être parfois étonnantes et mal considérées par les étudiants) ou des espaces francophones. Sans vouloir pour autant essentialiser l'autre dans cette rencontre, ni encore moins créer de processus d'étrangéisation, c'est la question des attitudes face à la diversité des pratiques professionnelles qui est en jeu. S'exposer à d'autres pratiques professionnelles permet un travail transversal sur l'habitus professionnel en construction, travail bénéfique pour toute la formation professionnelle. En contextualisant les situations de formation et en tenant compte de la complexité mais aussi de la multiplicité des individus et de leurs expériences, on tente alors non pas de figer ni de réifier les différences de pratiques professionnelles mais au contraire de les dépasser.

Si elles seules permettent de respecter la complexité du terrain, les pratiques réflexives, une fois institutionnalisées par la formation, présentent tout de même des limites :

La mise en scène de mea culpa orchestrés, de retours pseudo réflexifs où les étudiants sacrifiant à une contrition et à une auto-évaluation superficielles, voire factices, peut devenir une stratégie efficace pour contourner l'injonction à la réflexivité et tenter d'obtenir une « bonne » évaluation de la part des enseignants. (Goï, Huver, 2011, p. 203)

Ces limites invitent ainsi à une grande prudence lors de l'analyse des corpus recueillis dans le cadre de l'évaluation du dispositif.

\section{Préparation}

Des thèmes professionnels tels que les mesures disciplinaires, la relation adulte-enfant, l'espace scolaire ouvertfermé, l'organisation spatiale de l'école, la distance physique entre les acteurs scolaires, les rituels de classes, etc. sont abordés sur la base de témoignages authentiques d'anciens étudiants en stage ou de films documentaires. Chaque témoignage est soigneusement replacé dans son contexte social de production afin d'éviter de recréer des stéréotypes. Les étudiants sont censés alors prendre conscience de ce qui est construit chez eux et chez l'autre, le but n'étant pas tant de comprendre "pourquoi », mais de comprendre " comment " fonctionnent les mécanismes sociaux. Cette préparation contribue ainsi à développer chez eux un « regard anthropologique » (Berger, 2004).

\section{Accompagnement}

L'accompagnement prend la forme d'un forum interactif qui permet d'échanger entre pairs. Il est basé sur le concept du journal d'étonnement (Develotte, 2006). Les acteurs peuvent s'y étonner de ce qu'ils vivent et essayer d'objectiver ensemble le subjectif. Le forum est en ce sens un "dispositif de co-explicitation " avec les acteurs (Vinatier, 2009, p. 208).

\section{Analyse des expériences}

À leur retour, les étudiants sont invités à considérer les potentiels réinvestissements personnels et professionnels de leurs expériences à l'occasion lors du module obligatoire dont l'objectif est « de mettre vos expériences de mobilité au service de la formation professionnelle ${ }^{10}$. Ils analysent les potentiels réinvestissements personnels et professionnels de leurs expériences à l'occasion d'un module obligatoire. C'est ce réinvestissement qui est au cœur des préoccupations de formation. C'est d'ailleurs en se situant par rapport à leurs expériences que l'on peut

\footnotetext{
${ }^{10}$ II s'agit de nouveau d'une traduction personnelle du document officiel, disponible en ligne et rédigé en allemand (voir sitographie).
} 
prétendre les transformer en capital professionnel (Vinatier, 2009, p. 32). La mobilité est ainsi pensée comme lieu de formation professionnelle.

\section{Inviter les espaces francophones dans les cursus de formation}

\section{Le projet "Didactique de la mobilité » : de la diversité des pratiques scolaires}

Depuis l'été 2016, le projet de recherche-action-formation (RAF) intitulé «Didactique de la mobilité : pour un dispositif systémique de préparation, accompagnement et réinvestissement des expériences de formation en milieu francophone » a pour but de mettre au point un « dispositif ambitieux et complexe » (Molinié, 2015, p. 108) conçu pour encadrer les semestres d'échange universitaire des étudiants de toutes les formations initiales ${ }^{11}$. Fonctionnant en trois parties avant/pendant/après, il reprend en partie les conceptions théoriques et méthodologiques du nouvel encadrement des expériences de mobilité dans la formation initiale primaire ${ }^{12}$. Dans le cadre de cette RAF financée pour trois ans (2016-2019) ${ }^{13}$ par l'Institut für Forschung, Entwicklung und Evaluation de la PHBern, il est question d'évaluer les apports potentiels d'un tel dispositif, notamment en termes d'évolution des représentations envers le français. Ancrée en DLC mais empruntant largement à d'autres disciplines et notamment aux démarches socio-anthropologiques, notre approche méthodologique opère de constants aller-retours entre le terrain et les questionnements et est basée sur une co-construction avec les "informateurs ». Nous avons ainsi sélectionné cinq étudiants volontaires participant à un semestre d'échange et les avons suivis pendant trois semestres de leur formation au moyen d'une "multiplicité de corpus» (Robin, 2011) dont certains sont préconstitués (travaux institutionnels) et d'autres, provoqués dans le cadre de la RAF :

- Avant la mobilité - recueil en 2016

- fiche de données biographiques

- carte de langue(s) et de mobilité(s)

- Après la mobilité - recueil en 2017

- questionnaires

- travail de réflexion

- carte de langue(s) et de mobilité(s)

- entretien de groupe en auto-confrontation (Robin, 2015b, p. 292)

Les analyses qualitatives au moyen de catégories conceptualisantes (Paillé et Mucchielli, 2012) sont encore en cours $^{14}$ mais montrent déjà à ce stade que, véritable " distal » (Baudouin, 2009), la mobilité est vécue comme un moment fort de l'expérience de formation.

Fonctionnant sur l'axe paradigmatique du proche et du loin, c'est bien en se distanciant que les étudiants parviennent le mieux à analyser leur processus de formation : "Le système éducatif de la France [...] m'a aidée d'observer le système éducatif de la Suisse d'une autre et nouvelle façon " (Lisa) $)^{15}$. Le témoignage de cette étudiante montre par ailleurs que ses prédispositions favorables au développement d'un capital plurilingue et pluriculturel lui ont permis de faire face de manière détendue à l'inattendu et de tirer profit des rencontres: "Je ne

\footnotetext{
${ }^{11}$ Formations initiales des enseignants spécialisés, du primaire, du secondaire 1 et du secondaire 2.

${ }^{12}$ Les adaptations concernent le temps de mobilité (plus long, un semestre), la diversité des destinations et celle des formations initiales des participants.

${ }^{13}$ Rien n'indique à ce jour la volonté de l'institution de poursuivre la mise en place de ce dispositif au-delà de ces 3 ans de financement.

${ }^{14}$ II s'agit ici de la présentation de résultats partiels d'un projet en cours. Le développement des analyses croisées des apports de chaque type de corpus (ex: toutes les cartes de langue(s) et de mobilité(s)) et du profil de chaque informateur (chaque informateur en soi avec tous les corpus qu'il a co-construits) sont à suivre avec les actualités du projet sur: https://www.phbern.ch/didactique-de-la-mobilite-et-dispositif-daccompagnement-des-experiences-francophones-deformation/projekt.html

${ }^{15}$ Tous les prénoms sont fictifs.
} 
pensais pas que mon semestre d'échange à Toulouse me donnerait envie de voyager le monde arabe. C'est une surprise géniale». Lisa déclarait avant son départ que son objectif était de "rencontrer des Françaises », elle a finalement rencontré des personnes issues du " monde arabe » et a laissé cette altérité inattendue l'enrichir personnellement. Lisa explique que le dispositif a soutenu les développements qui se sont opérés chez elle: "j'aimerais bien vous remercier pour cette possibilité », nous écrit-elle dans son rapport.

Malgré l'initiation à l'anthropologie sociale ${ }^{16}$, certains, comme Peter, restent " coincés » sur le mode binaire du " eux vs nous »: "C'est plus ou moins le même contenu comme chez nous. C'est un peu plus scolaire que chez nous. On joue pas si beaucoup que chez nous ». Pour d'autres en revanche, l'évolution des représentations aussi bien que le développement des compétences professionnelles sont accrus en mobilité. Patricia raconte ses expériences de stage dans une école française :

J'ai essayé d'intégrer tous mes savoirs de la PH Berne, j'ai établi des préparations de cours avec beaucoup des changements de travail et des différents sou-sujets pour que ça soit plus intéressant. Mais déjà le premier jour j'ai remarqué qu'il y a beaucoup des différences et qu'il faut que j'adapte tous mes prépas ! Le niveau était moins fort que j'ai pensé et ils n'étaient pas du tout habitués aux travaux de groupes qui avait comme effet qu'ils n'ont pas travaillé et donc rien avancé. En plus je devais m'adapter aussi aux demandes du maître du stage car pour lui il y avait trop des changements pendant une leçon.

Elle prend conscience de ses propres " évidences" professionnelles (il faut varier les formes sociales au cours d'une leçon et changer fréquemment d'activité) et doit les expliciter et justifier auprès de praticiens étrangers qui ne semblent pas avoir incorporé les mêmes habitus professionnels :

Je suis toujours très curieuse pourquoi les enseignants font les choses d'une certaine façon alors je demandais et j'ai beaucoup discuté dans une bonne ambiance avec les profs. J'ai expliqué mon point de vue et ce que j'ai appris et donc pourquoi moi j'interviens comme ça. Comme ça je devais remettre en cause mes propres attitudes et argumenter. Mais ces différentes opinions sont très intéressantes.

À en juger par ces quelques lignes, cette nouvelle conception institutionnelle de la mobilité semble porter ses fruits : Patricia apprend à s'adapter à de nouvelles situations professionnelles, elle réajuste ses essais, analyse et échange. Dans son cas, la mobilité est le lieu de la professionnalisation par excellence. Elle semble être le complément idéal des enseignements théoriques reçus à la PHBern :

Grâce à ces choses que j'ai vues là-bas, je me suis rendu compte du système suisse et certaines choses qui sont traitées à la $\mathrm{PH}$ Berne. C'est par exemple la différence qui a manqué complètement chez l'enseignement de mon maître de stage. En effet, moi j'ai remarqué l'importance de celle-ci en voyant qu'il avait des élèves qui s'ennuyaient trop et ont donc commencé à faire des bêtises et des élèves qui étaient surmenées et qui ont perdu la motivation d’apprendre.

Ainsi la théorie est comprise et trouve une application concrète sur le terrain au contact de la diversité et du contre-exemple improbable en Suisse alémanique.

II reste toutefois malaisé pour la majorité des informateurs (à l'exception de Lisa) de situer le dispositif d'encadrement de leurs expériences dans leur parcours de formation professionnelle. Ainsi c'était "juste intéressant » pour Patricia, et surtout " c'était l'occasion de parler encore une fois, sur l'échange, mais maintenant c'est passé, alors, ça m'aide pas vraiment ". L'accent est mis avant tout sur la langue, sur l'opportunité d'utiliser le français alors même que lors des deux phases que sont l'accompagnement puis le réinvestissement, le français n'est pas la langue véhiculaire.

Selon cette même Patricia dont l'analyse des récits a révélé que la mobilité a été le lieu de la professionnalisation, les réinvestissements de la mobilité se situent simplement au niveau des compétences en langues: "on peut valoriser les connaissances en français, les connaissances de la langue ». Y aurait-il décalage entre les objectifs didactiques annoncés d'un côté et les expériences restituées dans les récits des acteurs ? Ou bien aurait-on affaire

\footnotetext{
${ }^{16}$ « la condition sine qua non pour construire la connaissance de l'autre et de soi » (Gohard-Radenkovic, 2016).
} 
à cette difficulté bien connue de dire les apprentissages, de se souvenir que l'on ne savait pas ${ }^{17}$, de réaliser ce et comment l'on a appris ? Le témoignage de Peter est exemplaire à ce titre : il déclare que le dispositif est superflu, "à mon avis, on n'a pas besoin de ça », alors même qu'il présente son rapport apaisé au français en fin de formation comme inné. Gommant tous les efforts d'apprentissage de la langue et toutes les difficultés liées à la mobilité auxquelles il a dû fait face, cette naturalisation du rapport au français est justement la preuve qu'il y a bien eu remédiation du rapport au français au travers de la mobilité. Ainsi la RAF semble indiquer à ce stade que, si le dispositif n'est pas forcément plébiscité par les acteurs ni par l'institution, il a un impact pourtant favorable sur leurs représentations du français.

\section{Le projet de formation bilingue au métier d'enseignant: de la diversité des pratiques institutionnelles}

Depuis 2016, la Haute École Pédagogique francophone des cantons de Berne, Jura et Neuchâtel (HEP-BEJUNE) et la PHBern travaillent à concevoir en partenariat une formation initiale des enseignants du primaire entièrement bilingue. La moitié de cette formation de trois années pour 180 ECTS aura lieu dès l'automne 2018 à la HEP-BEJUNE en français puis à la PHBern en allemand, donnant lieu à terme à un double diplôme des deux institutions ${ }^{18}$.

Basée sur le concept de "l'immersion réciproque ${ }^{19}$ la mise en place de cette formation bilingue exige une intense collaboration entre deux institutions, l'une alémanique et l'autre romande. Ces institutions ne se situant pas dans les mêmes villes et étant complètement indépendantes l'une de l'autre, les formateurs de part et d'autre ne $s^{\prime}$ étant jamais rencontrés ${ }^{20}$, réfléchir aux articulations possibles entre des programmes et des conceptions institutionnelles diverses était une gageure. II s'agit pour chacune des institutions de faire preuve d'ouverture et de respect envers le travail du partenaire et envers son programme de formation. À titre d'exemple, les pratiques scolaires suisses romandes décrites par Peter (« plus scolaire que chez nous, on joue pas si beaucoup ») font écho aux pratiques institutionnelles de formation des enseignants :

II y a beaucoup plus de stages que chez nous. Alors j'ai eu 6 semaines de stage pendant ce semestre. À mon avis c'est moins académique que chez nous, c'est une formation pour un métier, et pas une formation académique.

Paradoxalement, Peter nous décrit l'école romande comme plus académique que l'école alémanique alors que l'institution de formation le serait moins. Ces différences tant dans les pratiques scolaires (et donc les pratiques professionnelles de l'enseignement) que dans les pratiques institutionnelles de formation révèlent des différences de conception de l'éducation et du rôle de l'école. Ainsi, une telle collaboration de deux institutions relève de la prouesse au sein d'une Confédération où les plans d'études ne sont à l'heure actuelle pas (encore?) nécessairement les mêmes au sein d'un même canton (Berne par exemple) ni d'une même région linguistique ${ }^{21}$ : tolérance et décentration au contact de la diversité professionnelle sont de mises également pour les décideurs et l'ensemble des acteurs institutionnels. Le développement du " regard anthropologique " se joue autant au niveau des étudiants de cette future formation qu'au niveau des institutions et des rapports qu'elles entretiennent. Plus que jamais, l'initiation à l'anthropologie sociale semble nécessaire pour la réussite de l'entreprise.

Cette future formation témoigne d'une nouvelle aire à la PHBern : les espaces francophones sont non seulement reconnus, mais ils s'invitent même à présent au sein de l'institution alémanique. La mobilité sous toutes ses formes (géographique, linguistique, professionnelle, culturelle, virtuelle, etc.) et la formation professionnelle ne font

\footnotetext{
${ }^{17}$ Ces processus d'amnésie ne sont pas sans rappeler les effets de lissage de l’ « illusion biographique » (Bourdieu, 1986).

${ }^{18}$ Les diplômés auraient par la suite accès de plein droit aux postes d'enseignement primaire aussi bien en Suisse alémanique qu'en Suisse francophone ou bien encore au sein des quelques établissements bilingues.

${ }^{19}$ C'est-à-dire que les classes sont composées pour moitié d'étudiants francophones et pour moitié d'étudiants germanophones. L'enseignement est dispensé à parts égales en allemand et en français (Buser, 2014).

20 et ils ne connaissaient pas même le nom de leur homologue, les contacts entre homologues d'institutions de formation se faisant plutôt (à l'exception des institutions de formation bilingues) au sein des régions linguistiques respectives c'est-à-dire les $\mathrm{PH}$ alémaniques d'un côté, les HEP francophones de l'autre, quelle que soit la proximité géographique ou la délimitation cantonale.

${ }^{21}$ Plusieurs cantons alémaniques s'opposent au projet d'unification du Lehrplan 21.
} 
qu'un... sur le papier en tout cas. Quant au vécu des acteurs, il faudra encore attendre quelques années pour en savoir davantage sur leurs expériences.

\section{Conclusion : " change thinking, change practices " ?}

"Change thinking, change practices " ${ }^{22}$ ? Plusieurs chantiers pédagogiques appréhendant la pluralité linguistique et culturelle suisse s'ouvrent à l'heure actuelle à la PHBern. Tous ont en commun la conception du contact avec cette soi-disant « altérité francophone » et tournent autour de trois pôles : formation, mobilité, rapport à.

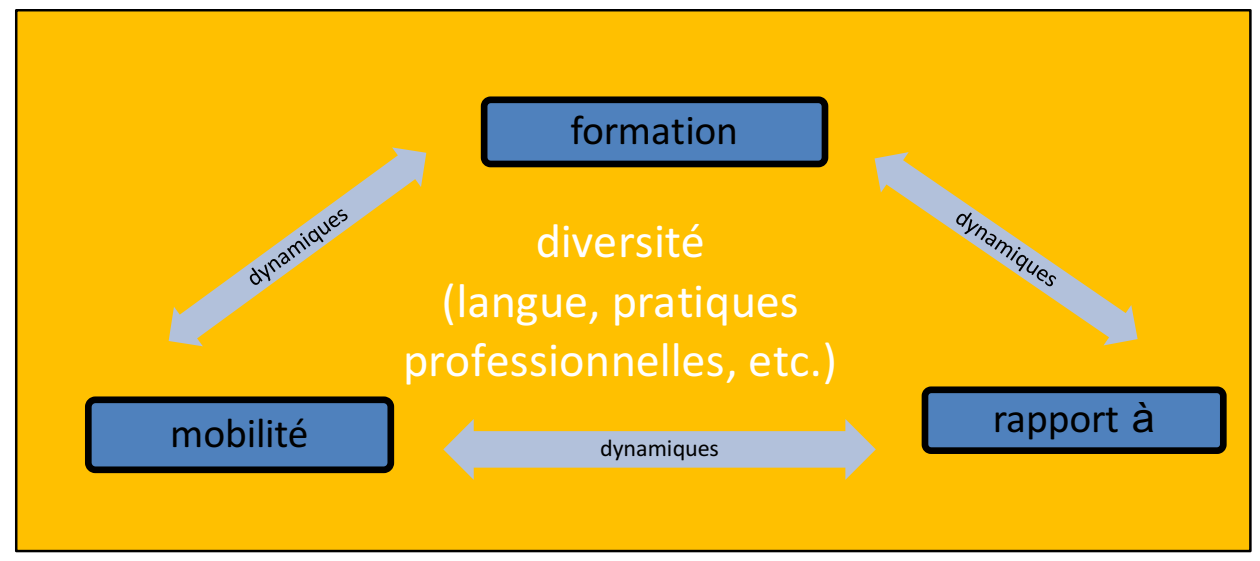

Figure 1 - Les pôles conceptuels communs aux projets

Ces projets inaugurent de prometteuses pistes didactiques qui tentent de dépasser la diversité des pratiques d'enseignement et des conceptions éducatives des deux communautés linguistiques. Ces changements de conceptions de la mobilité semblent indiquer que de nouvelles dynamiques se mettent en place, dont nous pouvons déjà évaluer en partie les répercussions sur la diversité des expériences individuelles de formation.

En guise de conclusion, nous émettons tout de même quelques doutes liés à notre position en tant qu'initiatrice des trois premiers projets présentés ci-dessus et en tant que coordinatrice du quatrième : ces dynamiques nous survivraient-elles? Si notre engagement personnel semble jusqu'à présent soutenu par les institutions concernées et que les discours affichés par les politiques éducatives cantonales le confirment, cela suffit-il à affirmer qu'il y a eu une réelle prise de conscience institutionnelle à la PHBern de la valeur formatrice des expériences professionnelles effectuées au contact d'autres espaces et une volonté de pérennisation de ce type de travail? Nous l'espérons...

\section{Références bibliographiques}

Auger, N., Molinié, M., Goï, C. et Guillaumin, C. (2011). Analyse de dispositifs universitaires FLE/S d'accompagnement au développement de compétences réflexives. Dans A. Bretegnier, Formation linguistique en contextes d'insertion. Compétences, posture, professionnalité: concevoir un cadre de référence(s) (p. 177-194). Berne : Peter Lang.

Anquetil, M. (2008). Apprendre à être étranger: des parcours de formation interculturelle pour les étudiants de mobilité. Dans F. Dervin et M. Byram, Échanges et mobilités académiques. Quel bilan? (p. 233-251). Paris : L'Harmattan.

Anquetil, M. (2012) La préparation linguistico-culturelle des étudiants Erasmus au départ pour la France: pour un projet d'interdidacticité franco-italienne. Repères-Dorif, Le français dans le contexte plurilingue des Centres Linguistiques Universitaires italiens. http://www.dorif.it/ezine/ezine_articles.php?art_id=9

\footnotetext{
${ }^{22}$ Titre de l'article de Trowler, Saunders et Kight (2004).
} 
Baudouin, J.-M. (2009). L'autobiographie à l'épreuve du texte. Dans D. Bachelart et G. Pineau (dir.). Le biographique, la réflexivité et les temporalités. Articuler langues, cultures et formation (p. 97-109). Paris : L'Harmattan.

Berger, C. (2004). Le travail critique du « regard anthropologique », partie prenante du processus d'évaluation. Dans G. Zarate et A. Gohard-Radenkovic, Les cahiers du Ciep. La reconnaissance des compétences interculturelles : de la grille à la carte, (p. 110-117). Paris : Didier.

Blanchet, P. (2016). Discriminations: combattre la glottophobie. Paris : Éditions Textuel.

Bourdieu, P. (1986). L’illusion biographique. Actes de la Recherche en Sciences Sociales, 62-63.

Buser, M. (2014). Deux plans d'études, une école : Scolarisation plurilingue dans la FiBi de Biel/Bienne. Une école publique basée sur le concept de l'enseignement par immersion réciproque. Enjeux Pédagogiques (HEPBEJUNE), 23, 31-36.

Carroll, R. (1987). Les évidences invisibles. Paris : Seuil.

Develotte, C. (2006). Le Journal d'étonnement. Aspects méthodologiques d'un journal visant à développer la compétence interculturelle (LIDIL- Rapport de stage et Mémoire professionnel, Univeristé de Grenoble). Grenoble : Presses Universitaires de Grenoble. https://lidil.revues.org/25

Gohard-Radenkovic, A. (2004). De la didactique d'une langue à la didactique des langues. Éducation et sociétés plurilingues, 17, 49-61.

Gohard-Radenkovic, A. (2009a). L'approche autobiographique dans la formation de futurs médiateurs linguistiques et culturels en situation de mobilité : de déplacements géographiques vers des déplacements identitaires. Dans A. Gohard-Radenkovic et L. Rachedi, Récits de vie, récits de langue et mobilités : Nouveaux territoires intimes, nouveaux passages vers l'altérité. Paris : L'Harmattan.

Gohard-Radenkovic, A. (2009b) Peut-on former à la différence ? Peut-on « penser la différence » dans la mobilité ? Les Cahiers de l'APLIUT - Pédagogie et recherche en Interculturel et enseignement des langues spécialisées, 28(2), 10-23. https://apliut.revues.org/3154

Gohard-Radenkovic (2016). Don et dette ou les fonctions du contre-don dans la construction des relations sociales. MediAzioni, 20. http://www.mediazioni.sitlec.unibo.it/images/stories/PDF_folder/document-pdf/202016/5\%20gohard.pdf

Goï, C. et Huver, E. (2011). La réflexivité comme compétence professionnelle en formation universitaire: une nécessité professionnelle ou une injonction (de) dans l'air du temps? Dans A. Bretegnier (dir.), Formation linguistique en contextes d'insertion. Compétences, posture, professionnalité: concevoir un cadre de référence(s) (p. 195-211). Berne : Peter Lang.

Laplantine, F. (1987). L'anthropologie. Paris : Seghers.

Molinié, M. (2015). Recherche biographique en contexte plurilingue, cartographie d'un parcours de didacticienne. Paris : Riveneuve.

Paillé, P. et Mucchielli, A. (2012). L'analyse qualitative en sciences humaines et sociales. Paris : Armand Colin.

Porcher, L. (1997). Lever de rideau. Dans G. Zarate (dir.), Les représentations en didactique des langues et cultures (p. 11-27). Paris : ENS de Fontenay/ Saint-Cloud.

Robin, J. (2011). Quand la multiplicité des questionnements se traduit par la multiplicité des corpus. Séminaire doctoral international INALCO - SOAS : Appropriation et transmission des langues et des cultures du monde, 66-71. https://www.soas.ac.uk/clp/doctoral-seminar/

Robin, J. (2013a). Français ? Où est le col ? Quand les étudiants suisses germanophones futurs enseignants du primaire doivent apprendre le français et effectuer un séjour de mobilité en région francophone.... Dans M. Vatz Laaroussi (dir.), Les défis de la diversité : enjeux épistémologiques, méthodologiques et pratiques (p. 119-128). Paris : L'Harmattan.

Alterstice - Revue Internationale de la Recherche Interculturelle, vol. $7, n^{\circ} 2$ 
Robin, J. (2013b). Séjour de mobilité linguistique obligatoire dans la formation des enseignants : oui, mais comment ? Le cas de la PHBern. Babylonia, 1, 94-98.

Robin, J. (2014a). Le rapport au français de futurs enseignants du primaire de la PHBern: récits d'expériences de formation et de mobilité (Thèse de doctorat, Université de Fribourg, Suisse). http://doc.rero.ch/record/210331

Robin, J. (2014b). Cartes de langue(s) et de mobilité(s) de futurs enseignants du primaire à Berne: quand une dynamique dialogique entre les corpus dévoile des représentations du français. Glottopol, Juillet, 64-79.

Robin, J. (2015a). Un semestre Erasmus comme « interstice institutionnel » ou quand le séjour de mobilité imposé par la formation des enseignants du primaire engendre des formes d'immobilités. Dans A. GohardRadenkovic et J. Veillette (dir.), Nouveaux espaces dans de nouvelles logiques migratoires? Entre mobilités et immobilités des acteurs (p. 201-223). Paris : Cahiers internationaux de sociolinguistique/L'Harmattan.

Robin, J. (2015b). "Ils aiment pas le français ". Le rapport au français de futurs enseignants du primaire de la PHBern dans leurs récits de formation et de mobilité. Berne : Peter Lang.

Trowler, P., Saunders, M et Knight, P. (2004). Change thinking, change practices. A guide to change for heads of department, subject centres and others who work middle-out.

https://www.heacademy.ac.uk/system/files/id262_change_thinking_change_practices.pdf

Vasseur, M.-T. (2008). Récits interactifs autour de la mobilité: partager, comprendre et analyser ensemble une expérience unique et commune. Dans F. Dervin et M. Byram (dir.), Échanges et mobilités académiques. Quel bilan? (p. 163-185). Paris : L'Harmattan.

Veillette, J., Gohard-Radenkovic, A. (2016). Culture, cultures... et interculturel. Dans C. Blons-Pierre \& P. Banon, Didactique du français langue étrangère et seconde dans une perspective plurilingue et pluriculturelle ( $p p$. 203-227). Berne, Peter Lang.

Vinatier, I. (2009). Pour une didactique professionnelle de l'enseignement. Rennes : Presses universitaires de Rennes.

Windisch, U. (2007). Le modèle suisse. Lausanne : L'Âge d’Homme.

Zarate, G. (1993). Représentations de l'étranger et didactique des langues. Paris : Didier.

\section{Sitographie}

Site Internet des « expériences de mobilité » de la PHBern, consulté le 11 mai 2017 : https://www.phbern.ch/studiengaenge/vp/studium/studienbereiche/fachwissenschaften-undfachdidaktiken/experiences-de-mobilite.html

Site Internet du projet de développement « Didactique de la mobilité » de l'Institut für Forschung, Entwicklung und Evaluation, consulté le 11 mai 2017 : https://www.phbern.ch/didactique-de-la-mobilite-et-dispositifdaccompagnement-des-experiences-francophones-de-formation/projekt.html 